ISSN 0103-5150

Fisioter. Mov., Curitiba, v. 24, n. 3, p. 535-547, jul./set. 2011 Licenciado sob uma Licença Creative Commons

\title{
Potencial de ação: do estímulo à adaptação neural
}

\author{
Action potential: from excitation to neural adaptation
}

\section{Eddy Krueger-Beck $^{[\mathrm{a}]}$, Eduardo Mendonça Scheeren ${ }^{[\mathrm{b}]}$, Guilherme Nunes Nogueira-Neto ${ }^{[\mathrm{c}]}$, Vera Lúcia da Silveira Nantes Button ${ }^{[\mathrm{d}]}$, Eduardo Borba Neves ${ }^{[\mathrm{e}]}$, Percy Nohama ${ }^{[\mathrm{f}]}$}

[a] Fisioterapeuta, MSc em Engenharia Biomédica pela Universidade Tecnológica Federal do Paraná (UTFPR), Doutorando em Engenharia Biomédica pela Universidade Tecnológica Federal do Paraná (UTFPR), Curitiba, PR - Brasil, e-mail: kruegereddy@gmail.com

[b] Educador físico, MSc em Ciências do Movimento Humano pela Universidade Federal do Rio Grande do Sul (UFRGS), Doutorando em Engenharia Biomédica pela Universidade Tecnológica Federal do Paraná (UTFPR), bolsista Capes, Curitiba, PR - Brasil.

[c] Engenheiro de computação, MSc em Engenharia Elétrica, Doutorando em Engenharia Biomédica pela Universidade Estadual de Campinas (Unicamp), bolsista CNPq, Campinas, SP - Brasil.

[d] Doutora, docente em Engenharia Biomédica pela Universidade Estadual de Campinas (Unicamp), Campinas, SP - Brasil.

[e] Doutor em Engenharia Biomédica pela Universidade Federal do Rio de Janeiro (COPPE/UFRJ), docente do Centro Universitário Campos de Andrade (Uniandrade), Curitiba, PR - Brasil.

[f] Doutor em Engenharia Biomédica pela Universidade Estadual de Campinas (Unicamp), docente da Pontifícia Universidade Católica do Paraná (PUCPR) e da Universidade Tecnológica Federal do Paraná (UTFPR), Curitiba, PR - Brasil.

\section{Resumo}

Introdução: 0 potencial de ação (PA) origina-se graças a uma perturbação do estado de repouso da membrana celular, com consequente fluxo de íons, por meio da membrana e alteração da concentração iônica nos meios intra e extracelular. Objetivos: Sintetizar o conhecimento científico acumulado até o presente sobre o potencial de ação neural e o seu processo de adaptação sob aplicação de um estímulo constante. Materiais e métodos: Busca realizada nas bases Springer, ScienceDirect, PubMed, IEEE Xplore, Google Acadêmico, Portal de Periódicos da Capes, além de livros referentes ao assunto. 0 idioma de preferência selecionado foi o inglês, com as keywords: action potential; adaptation; accommodation; rheobase; chronaxy; nerve impulse. Efetuou-se a procura de artigos com uma janela de tempo de 1931 a 2010 e livros de 1791 a 2007. 
Resultados: Dos trabalhos selecionados, foram extraídas informações a respeito dos seguintes tópicos: potencial de ação e suas fases; condução nervosa; reobase; cronaxia; acomodação; e adaptação neuronal. Conclusão: Um estímulo que crie PA, se aplicado de maneira constante, pode reduzir a frequência de despolarizações em função do tempo e, consequentemente, adaptar a célula. 0 tempo que a célula demora, na ausência de estímulos, para recuperar sua frequência original é definido como desadaptação.

Palavras-chave: Potenciais de ação. Adaptação fisiológica. Cronaxia. Tecido nervoso. Canais iônicos.

\begin{abstract}
Introduction: The action potential (AP) arises due to a disturbance of the resting state of the cell membrane with consequent flow of ions across the membrane and ion concentration changes in intra and extra cellular space. Objectives: This article aims to summarize the scientific knowledge accumulated to date on the action potential and neural adaptation in the process of applying a constant stimulus. Materials and methods: This is a literature review on the bases Springer, ScienceDirect, PubMed, IEEE Xplore, Google Scholar, Capes Periodicals Portal as well as books on the topic. The selected preferred language was English with the keywords: action potential; adaptation, accommodation; rheobase; chronaxy; nerve impulse. We conducted a search of articles with a wide time window from 1931 to 2010 and books from 1791 to 2007. Results: In the selected studies was extracted information about the following topics: action potential and its stages; nerve conduction; rheobase; chronaxie, accommodation, and adaptation. Conclusion: A stimulus that creates AP, if applied consistently, can reduce the frequency of depolarization as a function of time and, consequently, to adapt the cell. The time it takes the cell in the absence of stimuli, to recover its original frequency, is defined as a disadaptation.
\end{abstract}

Keywords: Action potentials. Physiological adaptation. Chronaxy. Nerve tissue. Ion channels.

\section{Introdução}

As células nervosas transmitem informações umas para as outras por meio de impulsos elétricos denominados potenciais de ação (PA) (1), tornando a comunicação dos neurônios similar a uma rede de circuitos eletrônicos $(2,3)$. PAs ocorrem o tempo todo em tecidos do corpo humano, coordenando suas funções, seja no estado de vigília, dormindo e em outros estados comportamentais (4). Com o uso de eletrodos de $\mathrm{Ag} / \mathrm{AgCl}(5)$ aderidos à superfície da pele ou microeletrodos conectados diretamente ao tecido nervoso(6), a atividade do PA pode ser mensurada por meio de várias técnicas de medição de sinal, tais como: eletro-oculografia (EOG), eletroencefalografia (EEG), eletroretinografia (ERG), eletrocardiografia (ECG), eletrococleografia (ECoG) e eletromiografia (EMG) (7). O PA gerado no interior do corpo produz um campo magnético perpendicular ao axônio, que pode ser registrado exteriormente por aparelhos como: magnetoencefalógrafo (MEG), magnetocardiógrafo (MCG) e o SQUID (acrônimo do inglês Super Conducting Quantum Interference Device) (8).
Historicamente, as primeiras descobertas sobre PA remontam a Claudius Galenus (129-199/217), que corroborava com os princípios aristotélicos que diziam que dos vasos sanguíneos do cérebro provinha a psychic pneuma (ou spiritus animalis, que hoje corresponde à atividade neural), conduzida pelos nervos até os músculos, que eram "inflados" gerando a contração. Após Galenus, foram criadas teorias similares, como a de René Descartes (1596-1650), que acreditava que os nervos movimentavam certo tipo de espírito por entre seus "tubos". Giovanni Alfonso Borelli (16081679), considerado o pai da biomecânica moderna, compactuava com a ideia de que um fluido, não um espírito, percorria os nervos e inchava os músculos provocando contração. William Gilbert (1544-1603) construiu o primeiro eletroscópio e Otto von Guericke (1602-1686) desenvolveu o primeiro gerador elétrico que produzia eletricidade estática (a partir de fricção) e que podia ser usado em experimentos com eletricidade. Esses instrumentos possibilitaram o desenvolvimento de estudos em eletrofisiologia (9).

Luigi Galvani (1737-1798) foi o primeiro a demonstrar, indiretamente, a propagação do PA em 
patas traseiras de rãs dissecadas (10). Seu sobrinho, Giovanni Aldini (1762-1834), foi o primeiro a aplicar estimulação elétrica em cadáveres frescos de criminosos executados. Postula-se que esse fato inspirou a escritora Mary Shelley (1797-1851) a escrever o livro Frankenstein ou o moderno Prometeu (11).

Johannes Peter Müller (1801-1858) afirmava que a duração do impulso nervoso era tão rápida que seria impossível mensurá-lo. Já seu discípulo, Emil Heinrich du Bois-Reymond (1818-1896), considerado por muitos como o pai da eletrofisiologia, refutando as ideias de seu professor, mostrou em 1843 a existência de uma flutuação negativa (do alemão negative schwankung) na tela do eletroscópio, durante uma tetania muscular produzida pelo uso do veneno estricnina $(9,11)$. Entre 1850 e 1852, Hermann von Helmholtz (1821-1894), colega de Bois-Reymond, determinou a velocidade de propagação do impulso nervoso, cálculo realizado a partir da distância entre os eletrodos e do tempo de atraso que precedia a contração muscular (11).

Um assistente de Helmholtz, Julius Bernstein (1839-1917), em torno de 1868, estudou a análise temporal do PA em ascendência, latência e descendência (9). 0 estudo revelou que o PA se move no sentido do corpo do neurônio (soma) para a extremidade do axônio (terminal pré-sináptico), denominada condução ortodrômica (1), que provém do grego orto (certo) e dromos (pista), presente na ativação eferente de fibras motoras e glandulares. Quando a condução ocorre da extremidade do axônio em sentido ao corpo do neurônio, denomina-se transmissão antidrômica (contra a pista), presente no sistema aferente sensitivo/sensorial (12).

Bernstein baseou-se no conceito de difusão de gases de Nernst para explicar o PA. Ele postulou que o PA ocorre em virtude da alta permeabilidade do $\mathrm{K}^{+}$ pela membrana celular comparado com outros íons, e pela maior concentração do íon no meio intracelular (MIC) em relação ao meio extracelular (MEC) (9). No ano de 1902, Bernstein propôs que durante o PA a célula nervosa adquiriria uma polaridade que não ultrapassava $0 \mathrm{mV}$ (13). Keith Lucas (1879-1916) determinou o "efeito tudo ou nada" do tecido muscular (14) e Edgar D. Adrian (1889-1977) descobriu que esse efeito também se aplicava ao tecido nervoso (15). O fisiologista francês Louis Lapicque (1866-1952) estudou a fundo os parâmetros necessários para a deflagração do PA em tecidos excitáveis em função da amplitude (reobase) e do tempo (cronaxia) (16).
O biofísico Alan Lloyd Hodgkin (1914-1998) e o fisiologista Andrew Fielding Huxley (1917-) desenvolveram, na década de 1940 , a técnica voltageclamp, que isola a membrana celular para mensurar o PA em axônio gigante de lula (Loligo brasiliensis). Posteriormente, em 1963, foram laureados com o neurofisiologista Sir John Carew Eccles (1903-1997) com o Prêmio Nobel de Fisiologia e Medicina (13). Hodgkin e Huxley (HH) corroboraram que o PA pode atingir no MIC uma amplitude aproximada de $40 \mathrm{mV}$ (13) e modelaram os canais iônicos presentes na membrana celular como voltagem-dependentes (17). Essa modelagem foi amplamente utilizada em muitos estudos posteriores (18), como em fibras mielinizadas de sapos (Xenopus laevis) (19), na membrana iônica dos neurônios auditivos da cóclea de mamíferos (20) ou mesmo para modelagens com uso de estimulação elétrica (21). Erwin Neher (1944-) e Bert Sakmann (1942-) realizavam experiências com a técnica patchclamp (22), isolando um único canal dependente de tensão elétrica de músculos denervados de sapos (23) e acabaram sendo laureados com o Prêmio Nobel de Fisiologia/Medicina em 1991.

O PA comporta-se de maneiras diferentes diante de diferentes estímulos físicos; quando um estímulo é muito lento, não rápido e intenso o suficiente para despolarizar a célula, acontece o fenômeno da acomodação $(24,25)$. A redução da frequência (de disparo da despolarização) após a exposição a um estímulo físico prolongado, como toque (26), alongamento muscular $(27,28)$, exposição a odores (29) e estimulação elétrica $(30,31)$, caracteriza a adaptação $(32$, 33). A recuperação da adaptação (desadaptação) ocorre após o cessar do estímulo, e demora até que a frequência dos estímulos volte a ser normal (34), salvo quando ocorre transdução genética, como na plasticidade sináptica $(14,35)$. 0 presente estudo trata de uma revisão da literatura sobre o desencadeamento e a propagação do PA e o consequente processo de adaptação celular.

\section{Materiais e métodos}

A busca de informações foi realizada nas bases Springer, ScienceDirect, PubMed, IEEE Xplore, Google Acadêmico e Portal de Periódicos da Capes e livros sobre o assunto. 0 idioma de preferência selecionado foi o inglês, com as keywords: action potential; adaptation; accommodation; rheobase; chronaxy; 
nerve impulse. Efetuou-se a procura de artigos com uma janela de tempo ampla, de 1931 a 2010, e livros de 1791 a 2007. Foram descartados três trabalhos relacionados à adaptação conhecida como plasticidade neuronal, por se entender que esse fenômeno está ligado a processos de aprendizagem e ocorre por estímulos repetidos e de longa duração, afastando-se do foco deste estudo. Após a realização das buscas nas bases de dados, foram lidos os abstracts e eliminadas as duplicações. Dos trabalhos selecionados, foram extraídas informações a respeito dos seguintes tópicos: potencial de ação e suas fases, condução nervosa, reobase, cronaxia, acomodação e adaptação neuronal.

\section{Resultados}

Foram selecionados os trabalhos que versavam sobre PA e o processo de adaptação celular. $\mathrm{O}$ artigo foi escrito a partir de 23 livros, 51 artigos encontrados no Google Acadêmico, 1 artigo do IEEE Xplore, 8 artigos da ScienceDirect e 9 artigos da base Springer, totalizando 92 referências.

Potencial de ação e suas fases

A fibra nervosa é classificada em função de seu diâmetro (Ø) e velocidade (V). Quanto maior o diâmetro, maior é a velocidade de condução nervosa. Joseph Erlanger (1874-1965) e Herbert Gasser (18881963), ganhadores do Prêmio Nobel de 1944 (14), apresentam a classificação dos tipos de fibra nervosa em função de seu diâmetro e velocidade de condução (Tabela 1). Como critério de comparação, o diâmetro médio do axônio gigante de lula é de 0,5 mm (13).

O PA pode propagar-se em dois sentidos principais: de fora do sistema nervoso central (SNC) em direção ao SNC (aferente) e vice-versa (eferente). Diversos são os mecanismos de deflagração do PA. Por exemplo, a ativação mecânica está presente na pressão sobre a pele (ou como toque e vibração sonora) e na pressão sanguínea sobre os barorreceptores localizados nas artérias carótidas. A ativação química está presente no paladar e olfato, enquanto o frio e o calor são mecanismos de ativação térmica. A ativação de mecanismos álgicos é decorrente de cortes e queimaduras, dentre outras situações que produzam dor. 0 mecanismo luminoso é ativado por fótons que incidem na retina. Todos os exemplos descritos fazem a transdução (37) desses fenômenos físicos em PAs $(36,38)$.

As membranas celulares são semipermeáveis (39) permitindo a passagem de apenas algumas substâncias como $\mathrm{Na}^{+}, \mathrm{K}^{+}, \mathrm{Cl}^{-}, \mathrm{Ca}^{++}$, proteínas e glicose, entre outras $(7,40)$. A Tabela 2 apresenta a concentração de $\mathrm{Na}^{+} \mathrm{K}^{+} \mathrm{e} \mathrm{Cl}^{-}$no MIC e MEC expressa em $\mathrm{mM}$ e $\mathrm{mEq} / \mathrm{L}$ de axônio gigante de lula (39).

Pela equação de Nernst (40), cada substância apresenta uma diferença de potencial entre MIC e MEC graças à diferença de concentração entre os meios (5). Em virtude dessa diferença de concentração e pelo efeito denominado equilíbrio de Gibbs-Donnan $(39,41)$, a membrana celular mantém-se polarizada. 0 potencial de repouso da célula é de aproximadamente $-70 \mathrm{mV}$ (Gráfico 1) $(19,28)$, encontrado por meio da equação $(1)(7,40)$ de Goldman-HodgkinKatz $(17,42)$, sendo T a temperatura em Kelvin; F o número de Faraday estipulado em $96.500 \mathrm{Cb} / \mathrm{mol}$; $\mathrm{P}_{\mathrm{x}}$ a permeabilidade de cada íon; e $\mathrm{R}$ a constante

Tabela 1 - Classificação da fibra nervosa em função do diâmetro e velocidade de condução

\begin{tabular}{lcc}
\hline Fibra nervosa & Diâmetro $(\mu \mathrm{m})$ & Velocidade $(\mathrm{m} / \mathrm{s})$ \\
\hline Aa & 15 & 100 \\
$A \beta$ & 8 & 50 \\
Ay & 5 & 20 \\
A $\delta$ & 3 & 15 \\
$B$ & 3 & 7 \\
C & 0,5 & 1 \\
\hline
\end{tabular}

Fonte: Adaptado de Schmidt et al. (36).

Tabela 2 - Concentração de sódio, potássio e cloro no meio intra e extracelular de axônio gigante de lula

\begin{tabular}{lrrrrr}
\hline & \multicolumn{3}{c}{ MIC } & & \multicolumn{2}{c}{ MEC } \\
\cline { 2 - 3 } \cline { 5 - 6 } & $\mathrm{mM}$ & $\mathrm{mEq} / \mathrm{L}$ & & $\mathrm{mM}$ & $\mathrm{mEq} / \mathrm{L}$ \\
\hline$\left[\mathrm{Na}^{+}\right]$ & 50 & 10 & & 440 & 142 \\
{$\left[\mathrm{~K}^{+}\right]$} & 400 & 140 & & 20 & 4 \\
{$\left[\mathrm{Cl}^{-}\right]$} & 52 & 4 & & 560 & 103 \\
\hline
\end{tabular}

Fonte: Adaptado de Schmidt et al. (36). 
universal dos gases, $8.314 \mathrm{~J} / \mathrm{mol} \mathrm{K}$. A equação de Goldman-Hodgkin-Katz foi confeccionada a partir da equação de Nernst (43).

$$
V_{m}=-\frac{R T}{F} \ln \left\{\frac{[\mathrm{Na}]_{i} P_{\mathrm{Na}}+[\mathrm{K}]_{i} P_{K}+[\mathrm{Cl}]_{i} P_{C l}}{[\mathrm{Na}]_{e} P_{\mathrm{Na}}[K]_{e} P_{K}+[C]_{e} P_{C l}}\right\}
$$

O PA ocorre na faixa de milissegundos (44), com frequências que variam de $20 \mathrm{~Hz}$ (1) até milhares de Hertz (36). Iniciado pela entrada abrupta do $\mathrm{Na}^{+}$no MIC, em virtude da diferença de concentração com o MEC, termina com a saída do $\mathrm{K}^{+}$e a consequente repolarização da célula (45). A diferença de concentração dos íons nos meios intra e extracelular pode ser modelada por meio de um circuito eletrônico como um capacitor, de aproximadamente 0,9 a 2 $\mu \mathrm{F} / \mathrm{cm}^{2}(6,45)$, que acumula energia e, em um determinado momento, descarrega-se por meio de um resistor (24) em paralelo; o MIC tem uma resistividade aproximada de 0,6 $\Omega . m$ (46) e a resistência da membrana celular varia entre 1 e $5 \mathrm{k} \Omega / \mathrm{cm}^{2}$ (6).

Todo íon tem o seu canal (47) específico na membrana permeável, sendo este dependente da diferença de potencial; outra substância, geralmente outro íon, funciona como bloqueador do canal. 0 precursor do PA na fibra nervosa é o íon $\mathrm{Na}^{+}$, tendo como bloqueador de seu canal o íon $\mathrm{Ca}^{++}(48)$. 0 canal iônico do $\mathrm{Na}^{+}$apresenta, segundo hipóteses de $\mathrm{HH}$, comportas de ativação (m, n e h), sendo a comporta h responsável pela inativação do canal $(33,49)$. Por possuir uma concentração mais baixa no MIC que no MEC (48), resultando em um gradiente de potencial elétrico (40) entre o MIC e MEC (50), o $\mathrm{Na}^{+}$tende a entrar, com isso, tem-se uma variação no potencial elétrico. 0 limiar de despolarização (LD) é de 15 $\mathrm{mV}$ em relação à tensão da membrana polarizada (Grafico 1); se essa variação ficar entre 10 e 15 mV ocorrerá um potencial graduado (45). 0 potencial graduado, local ou eletrotônico (36), percorre menos de $0,3 \mathrm{~mm}$ na fibra nervosa com rápida atenuação(3), retornando rapidamente ao potencial original da célula polarizada (51).

A corrente do íon $\mathrm{Na}^{+}$(19) é representada pela equação (2), sendo $\mathrm{IN}_{\mathrm{a}}$ a corrente de $\mathrm{Na}^{+}, \mathrm{gN}_{\mathrm{a}}$ a condutância ao $\mathrm{Na}^{+}, \mathrm{V}$ representa a tensão da membrana e $\mathrm{VN}_{\mathrm{a}}$ a tensão de $\mathrm{Na}^{+}$. A mesma modelagem matemática aplica-se para a corrente de $\mathrm{K}^{+}(24)$ como mostra a equação (3). Já a condutância ao íon $\mathrm{Na}^{+}$ (52) é expressa pela equação (4), onde $\mathrm{gN}_{\mathrm{a}} \mathrm{M}$ é uma constante; $\mathrm{m}^{3}$ representa uma comporta de ativação e h representa uma comporta de inativação (44).

$$
\begin{aligned}
& I_{N a}=g_{N a}\left(V-V_{N a}\right) \\
& I_{K}=g_{K}\left(V-V_{K}\right) \\
& g_{N a}=g_{N a M} \times m^{3} \times h
\end{aligned}
$$

Em um determinado momento o $\mathrm{Na}^{+}$quebra o bloqueio de $\mathrm{Ca}^{++}$e adentra abruptamente para o MIC, despolarizando a célula $(24,43)$ e aumentando a diferença de potencial da membrana a um platô de

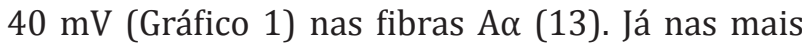
adelgaçadas, o platô não ultrapassa $0 \mathrm{mV}$. 0 valor entre $0 \mathrm{mV}$ e $+40 \mathrm{mV}$ é definido como overshoot (51). Na despolarização, a permeabilidade do $\mathrm{Na}^{+}$é 3,5 vezes maior que a permeabilidade do $\mathrm{K}^{+}$(19). Estudos recentes mostram que a corrente de $\mathrm{Na}^{+}$ é abrupta e com menos atraso em relação ao que postulavam HH (44); em neurônios centrais de mamíferos, a corrente de $\mathrm{Na}^{+}$é ativada sem atraso em comparação aos anfíbios (52). Em decorrência da corrente de $\mathrm{Na}^{+}$, a membrana celular apresentará uma variação entre $80 \mathrm{mV}$ (1) e $100 \mathrm{mV}$ (45) (dependendo do diâmetro da fibra), produzindo um período refratário absoluto (Gráfico 1) $(24,53)$ no qual um novo estímulo não conseguirá deflagrar outro PA nesse intervalo (efeito tudo ou nada) (51).

Após o período refratário absoluto, ocorre a saída do íon $\mathrm{K}^{+}$(54), onde a membrana eleva seu limiar de despolarização, necessitando de maior corrente para que ocorra uma nova despolarização (55). 0 processo de inativação reduz gradativamente a permeabilidade do $\mathrm{Na}^{+}$após ter ocorrido a despolarização da membrana, esse evento é conhecido como período refratário relativo (Gráfico 1) (24). 0 tempo de inativação estará quase completo quando a tensão na membrana for menor que $-30 \mathrm{mV}$ (24). Um pulso de $\mathrm{Na}^{+}$aplicado logo no início do período refratário relativo apresenta uma condutância de apenas 37\% quando comparado ao seu valor no período refratário absoluto. Entretanto, a tensão da membrana não sofre grande variação. Após 35 ms, a condutância do $\mathrm{Na}^{+}$retorna exponencialmente ao seu valor máximo (33). Em nervos mielinizados de coelhos, por exemplo, esse tempo de inativação é de duas a três vezes maior que em sapos (56).

Descobriu-se que a rápida entrada de $\mathrm{Na}^{+}$na célula reduz e atrasa o início da corrente de $\mathrm{K}^{+}$em 
axônios amielinizados das fibras musgosas do hipocampo de ratos, o que minimiza a sobreposição de seus respectivos influxos e difere dos apresentados por HH. Mesmo sendo em tecido amielinizado do SNC, os autores propõem que uma economia de processos neurais está associada à evolução (57). A duração do PA não é a mesma para todos os neurônios. Por exemplo, os neurônios que secretam dopamina têm um período refratário relativo maior que as células piramidais CA1 do hipocampo e maior ainda quando comparado às células de Purkinje do cerebelo (51).

Ao final da saída dos íons $\mathrm{K}^{+}$ocorre o intumescimento da célula em virtude do acúmulo de ânions no MIC (58). Ocorre a ativação da bomba de $\mathrm{Na}^{+}-\mathrm{K}^{+}$ATPase $(28,40)$, que contribui para a manutenção do potencial de repouso da célula (59), liberando três íons de $\mathrm{Na}^{+}$para o MEC a cada dois íons de $\mathrm{K}^{+}$para o MIC (48). 0 afluxo de ânions para o MIC gera hiperpolarização, deixando o MIC mais negativo que o rotineiro, definindo o estado ulterior negativo, sendo mais difícil de despolarizar a célula nesse momento (50). Consequentemente, ocorre o estado ulterior positivo, ocasionando um leve aumento na tensão da membrana no MIC, antes que retorne ao seu estado de polarização original (38). Os eventos que compreendem o PA estão ilustrados no Gráfico 1.

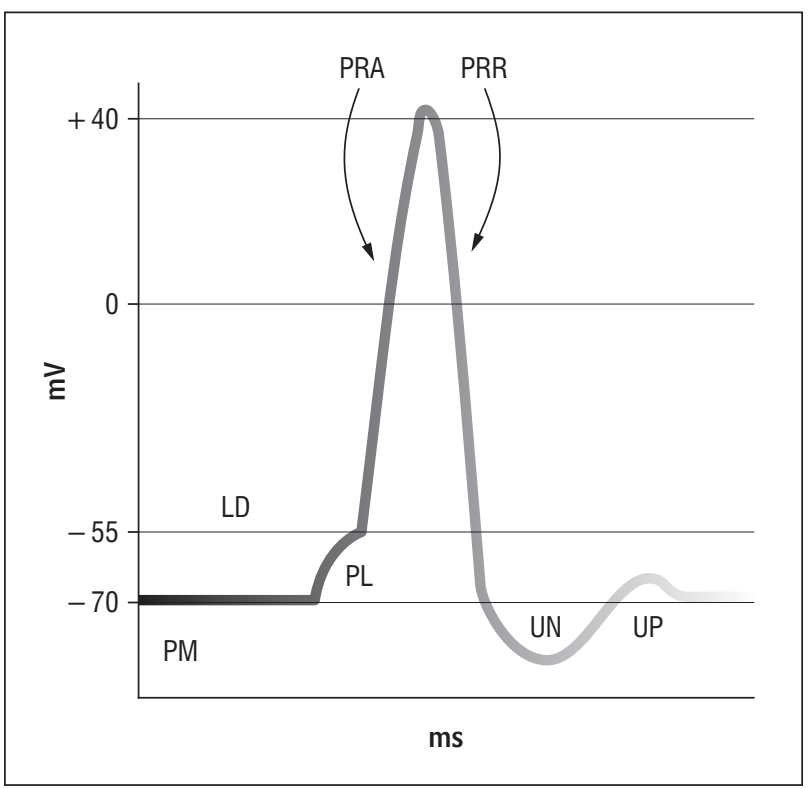

Gráfico 1 - Potencial de ação e suas fases

Legenda: $\mathrm{mV}=10-3 \mathrm{~V} ; \mathrm{PM}=$ potencial de membrana; $\mathrm{LD}=$ limiar de despolarização; PL = potencial local; PRA = potencial refratário absoluto; $\mathrm{PRR}=$ período refratário relativo; $\mathrm{UN}=$ estado ulterior negativo; UP = estado ulterior positivo.

Fonte: Dados da pesquisa.

\section{Condução nervosa}

Em um sinal eferente, o PA origina-se no cone do axônio (3), que é o corpo do neurônio adelgaçandose no axônio. A condução nervosa ocorre de maneira saltatória $(58,60)$ nas fibras mielinizadas, por meio da despolarização dos nódulos de Ranvier (NR) (61). A função da mielina é servir como um isolante elétrico aumentando a velocidade da condução nervosa (48). 0 PA também pode ser deflagrado quando uma estimulação elétrica é aplicada no internódulo, porém, o PA terá menor amplitude. Em nervos isquiáticos de rãs, Huxley e Stämpfli (1949) mostraram que a amplitude do PA reduz à medida que se aproxima do internódulo e aumenta quando se aproxima do nódulo, chegando ao seu máximo ao atingi-lo. O PA sofre um atraso no NR graças ao fato de que essas regiões apresentam condutância e capacitância maiores que nos internódulos. Internamente, não existe atraso na geração do PA em um internódulo; já quando atingem o NR, ocorre um pequeno atraso na geração do PA (60).

A partir da modelagem matemática da membrana da célula nervosa, constatou-se que o axônio gigante de lula não é um bom condutor de eletricidade (51). Sugeriu-se, então, que modelos como o de $\mathrm{HH}$, entre outros, deveriam ser revisados. Face ao estudo realizado, a forma mais indicada deveria estar de acordo com a equação [5], onde D pode ser considerado como a dimensão fractal (em vários módulos) da sessão do axônio e A é a área do condutor (62).

$$
R \sim \frac{1}{A^{D /(D+1)}}
$$

O PA dura poucos milissegundos, mas em casos particulares pode durar até dias em razão das alterações bioquímicas, como da proteína G no MIC. Um exemplo desse tipo de receptor é o N-methyl-daspartate (NMDA) do neurotransmissor glutamato (63), localizado na Cornu Ammonis (50) do hipocampo, região encefálica correspondente à memória $(55,64)$.

\section{Reobase, cronaxia e acomodação}

Reobase é o estímulo elétrico de amplitude mínima (25) para despolarizar uma célula excitável (12, 
49), quando se aplica um pulso de longa duração (300 ms). Cronaxia é definida como a menor duração de pulso para deflagrar um PA (5), com um estímulo configurado com o dobro da amplitude da reobase. 0 diâmetro do axônio é inversamente proporcional à cronaxia, assim, quanto maior o diâmetro do axônio, menor a duração do pulso elétrico necessário para despolarizar a célula (65). Já a reobase é inversamente proporcional à duração do pulso, sendo necessária uma amplitude de estímulo maior quanto menor for a duração do pulso (5).

A acomodação decorre do incremento lento de amplitude (ao nível sublimiar) (5) em função do tempo, fazendo com que a célula aumente o seu LD (66) gerando apenas um potencial graduado e não um PA (67). Estudos em sapos indicaram que os gânglios espinais possuem rápida acomodação, necessitando de um incremento de amplitude muito rápido para evitar-se acomodação, ao passo que os gânglios simpáticos apresentam menor grau de acomodação e os motoneurônios muito menos dependência de um incremento rápido na amplitude para evitar a acomodação (25). 0 baixo grau de acomodação em motoneurônios possivelmente deve-se à dependência da troca de condutância do $\mathrm{K}^{+}$na fase de inativação do $\mathrm{Na}^{+}$.

\section{Adaptação neuronal}

Diferentemente da acomodação que ocorre antes da primeira despolarização da célula, um estímulo físico específico, aplicado sobre um receptor neuronal, logo alcança o seu platô e tem sua frequência de PAs reduzida em função do tempo (68), tanto no tecido nervoso periférico (66) como central (69). A sensibilidade (37) de uma célula nervosa diante de um estímulo contínuo ou repetitivo (constante dentro de um padrão) é definida como adaptação ou habituação (42), decorrente do aumento do seu limiar de despolarização (29) sem deflagrar novos PAs, como ilustra o Gráfico 2. 0 termo em inglês "spike frequency adaptation" é utilizado por alguns autores com o significado de adaptação $(70,71)$.

Logo após a aplicação de um estímulo elétrico constante para a despolarização da célula nervosa, existe uma rápida queda $(\sim 0,1 \mathrm{~s})$ na frequência de despolarização, denominada de adaptação inicial. Após esse evento, a frequência começa a decair lentamente de forma exponencial, caracterizando a adaptação

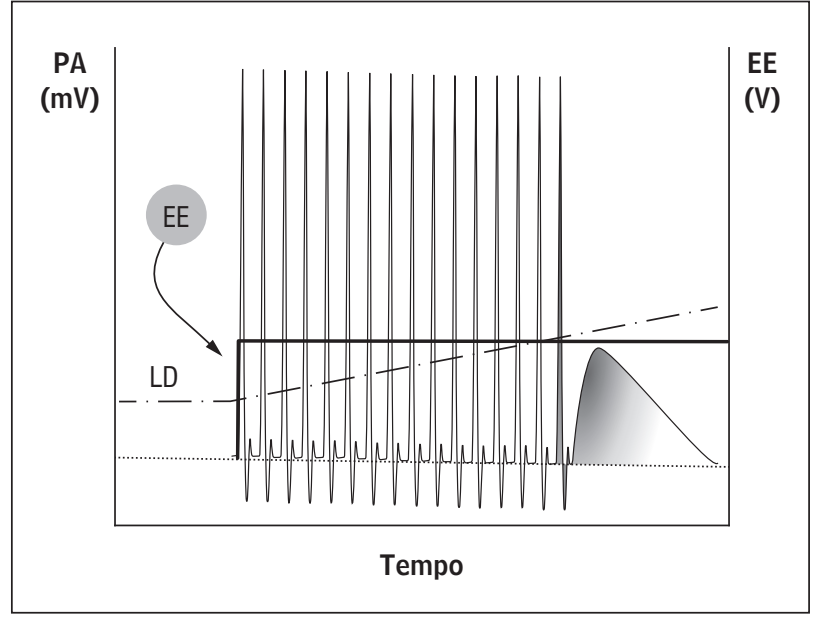

Gráfico 2 - Adaptação do potencial de ação sobre um estímulo prolongado

Legenda: PA = potencial de ação; $E E$ = estímulo elétrico (traço cheio); LD = limiar de despolarização (linha com traço e ponto), linha tracejada representa o potencial de repouso do PA e a ausência de estimulação elétrica.

Fonte: Dados da pesquisa.

tardia (72). A adaptação inicial do motoneurônio pode ocorrer graças ao aumento da condutância do $\mathrm{K}^{+}$(73), enquanto a adaptação tardia parece estar ligada à inativação dos canais iônicos de $\mathrm{Na}^{+}(33$, 66). 0 bloqueio bioquímico da bomba de $\mathrm{Na}^{+}-\mathrm{K}^{+}-$ ATPase não influencia na adaptação do motoneurônio em qualquer fase (72).

Os corpúsculos de Paccini são exemplos de receptores de rápida adaptação (66), as terminações de Ruffini são de adaptação lenta (74), assim como os nociceptores (38), de temperatura (68) e adaptação em receptores olfatórios, que são ativados por meio de substâncias químicas (75). A recuperação da adaptação (desadaptação) corresponde ao intervalo de tempo após a adaptação no qual a célula não sofre qualquer estímulo e retorna ao seu limiar de despolarização original (29). A variação da amplitude do estímulo apresenta-se como forma de retardar a adaptação (68).

A adaptação existe em mecanoceptores $(32,76)$ como o órgão tendinoso de Golgi (28). No fuso muscular, as fibras anuloespirais (primárias) da bolsa nuclear (tipo Ia), que registram o comportamento dinâmico do músculo (32), são de rápida adaptação. Já as fibras secundárias (tipo II), que medem o músculo de forma estática, são de adaptação lenta (55) graças ao fato de serem utilizadas por longo tempo, como os músculos responsáveis por manterem o 
corpo humano em posição ortostática. No fuso muscular do sapo (77), o alongamento muscular em função do tempo resulta em diminuição da frequência de PA $(78,79)$.

Estudou-se a adaptação de nervos cutâneos de sapos diante da estimulação proporcionada por jatos de ar. Decorridos 22s após o estímulo, a frequência de impulsos decaía mais da metade, de aproximadamente $150 \mathrm{~Hz}$ para $60 \mathrm{~Hz}$ (26). Gray e Matthews (1951) estudaram a adaptação de corpúsculos de Paccini do mesentério de gatos. Mostraram que a fibra nervosa, exposta a um estímulo mecânico que aumenta linearmente, adapta-se muito mais rapidamente quando comparada a um estímulo elétrico aplicado da mesma maneira sobre o nervo correspondente ao corpúsculo de Paccini (27). Esses resultados mostram que diferentes estímulos físicos têm respostas de adaptação diferenciadas sobre o mesmo receptor.

Por meio do músculo estriado esquelético ex vivo de gato, relacionou-se o nível de tensão de estimulação elétrica, que não deflagrasse uma contração muscular, com o alongamento mecânico resultante para verificar a resposta do fuso muscular. Com a estimulação elétrica, a frequência do fuso muscular manteve-se em $27 \mathrm{~Hz}$ com variação de $10 \%$ do valor inicial, durante todo o experimento $(1,4 \mathrm{~s})$. Com o alongamento muscular, o receptor alcançou inicialmente $92 \mathrm{~Hz}$ e em $0,3 \mathrm{~s}$ decaiu para $25 \mathrm{~Hz}$ e se manteve durante o resto do tempo $(1,4 \mathrm{~s}$ de tempo total). Esses resultados tiveram um comportamento similar em todas as amostras $(\mathrm{N}=20)(31)$. E sugerem um comportamento diferenciado do fuso muscular em relação ao comprimento muscular.

Em estudo com células olfatórias, demonstrouse que o tempo de adaptação e desadaptação varia de $500 \mathrm{~ms}$ até $10 \mathrm{~s}$, criando uma analogia com a eletrônica de uma banda passante (faixa de atuação do sinal) (80) de $500 \mathrm{~ms}$ a $10 \mathrm{~s}$. A equação (6) reproduz esse tipo de adaptação, onde $\mathrm{WT}_{\mathrm{a}}$ é o peso da função (porcentagem do aumento do limiar de despolarização), $\mathrm{T}_{\mathrm{a}}$ representa a fase de adaptação, t é tempo de início do estímulo até o ponto de maior aumento do limiar de despolarização. Esse limiar eleva-se exponencialmente. Para a desadaptação, utiliza-se a equação (7).

$$
W_{T a}=\frac{0.55 \times 1.5^{(0.1 \times t)}}{\sum W_{T a}}
$$

$$
W_{T d}=\frac{0.55 \times 1.5^{[0.1 \times(T-t)]}}{\sum W_{T d}}
$$

Sendo $W_{\text {Td }}$ o peso da função em relação ao retorno do limiar de despolarização ao seu valor original (em porcentagem a partir do valor no início da desadaptação), T o período da amostra (desde o início do estímulo) e t o tempo a partir do tempo máximo de $\mathrm{T}_{\mathrm{a}}$ da equação [6]. 0 tempo de retorno da adaptação decai exponencialmente em função do tempo, conforme Moore (29).

Em outro estudo, estimulou-se eletricamente com frequências de 4 e $8 \mathrm{~Hz}$ numa e intensidades na faixa de 0,1-0,5 mA sobre o rostro de roedores (próximo à vibrissa) (30). 0 tempo de adaptação no núcleo ventral póstero-medial (NVPM) (81) do tálamo foi medido e comparado ao tempo de adaptação das células em barril do córtex somatossensorial primário (81). Com $4 \mathrm{~Hz}$ em $5 \mathrm{~s}$ de aplicação, o núcleo ventral póstero-medial apresentou adaptação da amplitude que reduzia de $31 \% \pm 9 \%$ em comparação aos $79 \% \pm 6 \%$ do córtex somatossensorial primário. O NVPM tem um tempo de desadaptação médio de $5 \mathrm{~s}$ para retornar ao valor de $95 \%$ (valor normalizado em 100\%); já o SI demorava 13s para obter o mesmo efeito. Com estímulos elétricos em $8 \mathrm{~Hz}$ e 2,5s, o núcleo ventral póstero-medial mostrou uma redução de $70 \% \pm 6 \%$ de sua amplitude e o SI $93 \% \pm 5 \%$, com um tempo de recuperação muito rápido (para atingir os $95 \%$ ) no núcleo ventral póstero-medial (aproximadamente 1s) contra aproximadamente $22 \mathrm{~s}$ no córtex somatossensorial primário. A tensão da membrana cortical não sofreu variação no estado polarizado, apresentando $-61,7$ $\pm 1,3 \mathrm{mV}$ previamente à estimulação, contra $-62,1$ $\pm 1,4 \mathrm{mV}$ logo após a adaptação, mostrando que a hiperpolarização não ocorre em nível cortical (30).

A adaptação dos motoneurônios e dos interneurônios em tartarugas com aplicação da estimulação elétrica durante 30 s é descrita de modo similar na literatura. Nos primeiros $5 \mathrm{~s}$ de estímulos, a frequência decai em torno de 33\%, e depois continuava decaindo durante os próximos $25 \mathrm{~s}$, até atingir uma redução em frequência de aproximadamente $40 \%$ (82). A adaptação do motoneurônio tem um decaimento exponencial, sendo modelada pela equação (8):

$$
f=k+A_{1} e^{-t / \tau 1}+A_{2} e^{-t / \tau 2}+\ldots+A_{n} e^{-t / \tau n}
$$


Onde $t$ representa tempo (em segundos) após o primeiro spike do sinal, $k$ é constante de equilíbrio (quando a frequência passa de um exponencial para outra), $A_{1} \ldots A_{\mathrm{n}}(\mathrm{Hz})$ são as amplitudes de cada exponencial, e $\tau 1$ e $\tau n$ são constantes de tempo, conforme Gorman et al. (82).

Em sanguessugas, estimulando eletricamente o motoneurônio, independente da intensidade aplicada $(0,5 ; 1 ; 1,5 ; 2 \mathrm{nA})$, a frequência de despolarização do sinal decaía $50 \%$ em 1s após a aplicação (83). Na adaptação de motoneurônios do nervo hipoglosso de ratazanas durante a aplicação de estimulação elétrica constante de $60 \mathrm{~s}$, ocorria uma redução de $\sim 30 \mathrm{~Hz}$ para $\sim 22 \mathrm{~Hz}$ nos primeiros $10 \mathrm{~s}$ de estimulação, e depois a frequência continuava a decair suavemente nos 50 s restantes (72).

0 músculo estriado esquelético é dividido basicamente em fibras do tipo F (ou II) de rápida contração/fadiga muscular e do tipo S (ou I) de lenta contração/fadiga muscular (84). Em contração isométrica, as frequências de disparo dos motoneurônios ficam entre $20 \mathrm{~Hz}$ e $50 \mathrm{~Hz}$; fibras musculares com maior número de fibras $\mathrm{S}$ apresentam menores frequências; o contrário ocorre com as fibras F (85). Na contração isométrica, o intervalo de pulso é incrementado, reduzindo, assim, a frequência do sinal (86). A adaptação é mais pronunciada em motoneurônios de fibras $\mathrm{F}$ do que S (87). Em humanos, com uma contração voluntária sustentada durante $60 \mathrm{~s}$, a frequência do motoneurônio decai de $27 \mathrm{~Hz}$ para $14 \mathrm{~Hz}$ (88). A inibição central provocada pela célula de Renshaw (inibitória) na medula espinal pode influenciar no declínio da frequência do motoneurônio durante a fadiga muscular (87) e não somente por fatores da fibra nervosa em atividade.

Usando eletrodo intracelular localizado no músculo gastrocnêmio medial de gatos anestesiados, aplicou-se estimulação elétrica com frequência de burst em $40 \mathrm{~Hz}$, durante um período de $4 \mathrm{~min}$, continuamente; o sinal foi adquirido nos motoneurônios. Demonstrou-se que a aplicação do estímulo prolongado fez com que a frequência do PA tivesse um decremento de aproximadamente $50 \%$ nos 30 s iniciais, quando então começava a decair muito lentamente até o final dos $4 \mathrm{~min}$, sem alteração da amplitude do sinal (89). Repetindo o mesmo estudo, mas com diferenciação entre os tipos de fibras musculares, observou-se que as fibras $\mathrm{F}$ apresentavam decaimento da frequência de disparo mais rapidamente que as fibras S. Com os valores normalizados, para o primeiro minuto de estimulação, a adaptação das fibras rápidas provocou uma queda de $60 \%$ da força, acompanhando o declínio da frequência; o mesmo não ocorreu com as fibras S (90) e tanto a frequência como a força variaram muito pouco no primeiro minuto. Por meio da eletromiografia, demonstrou-se que em contração voluntária de músculo deltoide existe uma forte correlação negativa entre o disparo do motoneurônio e o desenvolvimento de força na fibra muscular (91). Novamente com gatos anestesiados, avaliou-se a adaptação dos motoneurônios de músculos do tipo $\mathrm{F}$ e $\mathrm{S}$ diante de uma estimulação elétrica constante. Durante a estimulação elétrica mantida durante 240 s, um motoneurônio tipo $\mathrm{F}$ apresentou redução de $\sim 30 \mathrm{~Hz}$ para $\sim 3 \mathrm{~Hz}$ ao final do período de estimulação, enquanto o motoneurônio de uma fibra tipo $\mathrm{S}$ apresentou redução de $\sim 20 \mathrm{~Hz}$ para $\sim 15 \mathrm{~Hz}$ ao final do experimento (92), demonstrando que a redução da frequência do motoneurônio é análoga à fadiga muscular.

\section{Conclusão}

O potencial de ação representa o meio de comunicação do tecido nervoso com outros tipos de tecidos, em funções como coordenar um movimento ou secretar um hormônio na circulação sanguínea, dentre outras tantas atividades. Vários são os estágios que compreendem a geração do potencial de ação, podendo este ser eliciado a partir de vários estímulos físicos. Estímulos que criem PAs são aplicados de maneira constante, reduzem a frequência de despolarização em função do tempo e, consequentemente, levam a célula à adaptação, diferenciando-se do evento de acomodação, em que o limiar de despolarização eleva-se antes do primeiro potencial de ação.

A elevação do limiar de despolarização apresentase como uma das condições que geram a adaptação do potencial de ação. Por intermédio de modelagens matemáticas, pode-se formular o resultado da adaptação sobre um determinado tecido nervoso e sua futura desadaptação. 0 fenômeno da adaptação também ocorre nos fusos musculares e motoneurônios durante a estimulação elétrica, podendo ser percebido em conjunto com a fadiga muscular, graças à relação intrínseca existente entre fadiga muscular e adaptação do motoneurônio. Há uma enorme dificuldade em se relacionar a resposta motora com um dos dois eventos, tendo em vista a sua complexibilidade. 
A adaptação do neurônio sob a aplicação de estímulos constantes tem elevado potencial de aplicação na reabilitação de portadores de lesões neurológicas. A aplicação desse conhecimento em sistemas de estimulação elétrica funcional, por exemplo, contribui na elaboração de estratégia de controle do estimulador (sistema em malha fechada).

\section{Agradecimentos}

Os autores gostariam de agradecer à Capes (mestrado e doutorado) e ao CNPq (doutorado) pelas bolsas concedidas para a realização deste trabalho.

\section{Referências}

1. Popovic MR, Thrasher TA. Neuroprostheses. In: Bowlin GL, Wnek G, editors. Encyclopedia of biomaterials and biomedical engineering. New York: Informa Healthcare; 2004. p. 1056-65.

2. Laughlin SB, Sejnowski TJ. Communication in neuronal networks. Science. 2003;301(5641):1870-4.

3. Marmarelis VZ. Signal transformation and coding in neural systems. IEEE Trans Biomed Eng. 1989;36(1): 15-24.

4. Sheeba V, Gu H, Sharma VK, O'Dowd DK, Holmes TC. Circadian-and light-dependent regulation of resting membrane potential and spontaneous action potential firing of Drosophila circadian pacemaker neurons. J Neurophysiol. 2008;99(2):976-88.

5. Merrill DR, Bikson M, Jefferys JGR. Electrical stimulation of excitable tissue: design of efficacious and safe protocols. J Neurosci Methods. 2005; 141(2):171-98.

6. DiLorenzo DJ, Bronzino JD. Neuroengineering. Boca Raton, FL: CRC Press; 2007.

7. Northrop RB. Analysis and application of analog electronic circuits to biomedical instrumentation. Boca Raton, FL: CRC; 2004.

8. Barnes FS, Greenebaum B. Handbook of biological effects of electromagnetic fields. 3rd ed. Boca Raton, FL: CRC; 2006.

9. Bennett MR. The early history of the synapse: from Plato to Sherrington. Brain Res Bull. 1999;50(2): 95-118.
10. Galvani L. De viribus electricitatis in motu musculari commentarius. Bon Sci Art Inst Acad Comm. 1791;363-418.

11. Verkhratsky A, Krishtal OA, Petersen OH. From Galvani to patch clamp: the development of electrophysiology. Pflugers Arch. 2006;453(3):233-47.

12. Robinson AJ, Snyder-Mackler L. Eletrofisiologia clínica: eletroterapia e teste eletrofisiológico. 2 a ed. Porto Alegre: Artmed; 2001.

13. Mudado MA, Moreira TH, Cruz JS. $\mathrm{O}$ início da era dos canais iônicos. Ciência Hoje. 2003;33(193):56-8.

14. Cooper SJ. Donald O. Hebb's synapse and learning rule: a history and commentary. Neurosci Biobehav Rev. 2005;28(8):851-74.

15. Adrian ED. The all-or-none principle in nerve. J Physiol. 1914;47(6):460-74.

16. Brunel N, van Rossum MCW. Lapicque's 1907 paper: from frogs to integrate-and-fire. Biological Cybernetics 2007;97(5):337-9.

17. Hille B. Ionic channels of excitable membranes. 2nd ed. Sunderland: Sinauer; 1991.

18. Rudolph M, Pospischil M, Timofeev I, Destexhe A. Inhibition determines membrane potential dynamics and controls action potential generation in awake and sleeping cat cortex. J Neurosci. 2007;27(20):5280-90.

19. Frankenhaeuser B, Huxley AF. The action potential in the myelinated nerve fibre of Xenopus laevis as computed on the basis of voltage clamp data. J Physiol. 1964;171(2):302-15.

20. Cartee LA. Evaluation of a model of the cochlear neural membrane. II: comparison of model and physiological measures of membrane properties measured in response to intrameatal electrical stimulation. Hearing Research. 2000;146(1-2):153-66.

21. Jiang W, Tsang KM, Hua Z. Hopf bifurcation in the Hodgkin-Huxley model exposed to ELF electrical field. Chaos, Solitons and Fractals. 2004;20(4):759-64.

22. Sakmann B, Neher E. Patch clamp techniques for studying ionic channels in excitable membranes. Annu Rev Physiol. 1984;46(1):455-72.

23. Neher E, Sakmann B. Single-channel currents recorded from membrane of denervated frog muscle fibers. Nature. 1976;260(5554):799-802. 
24. Hodgkin AL, Huxley AF. A quantitative description of membrane current and its application to conduction and excitation in nerve. J Physiol. 1952;117(4): 500-44.

25. Stoney SD, Jr, Machne X. Mechanisms of accommodation in different types of frog neurons. J Gen Physiol. 1969;53(2):248-62.

26. Cattell MK, Hoagland $\mathrm{H}$. Response of tactile receptors to intermittent stimulation. J Physiol. 1931;72(4): 392-404.

27. Gray JAB, Matthews PBC. A comparison of the adaptation of the Pacinian corpuscle with the accommodation of its own axon. J Physiol. 1951;114(4):454-64.

28. Fukami Y. Responses of isolated Golgi tendon organs of the cat to muscle contraction and electrical stimulation. J Physiol. 1981;318(1):429-43.

29. Moore PA. A model of the role of adaptation and disadaptation in olfactory receptor neurons: implications for the coding of temporal and intensity patterns in odor signals. Chem Senses. 1994;19(1):71-86.

30. Chung S, Li X, Nelson SB. Short-term depression at thalamocortical synapses contributes to rapid adaptation of cortical sensory responses in vivo. Neuron. 2002;34(3):437-46.

31. Lippold OCJ, Nicholls JG, Redfearn JWT. Electrical and mechanical factors in the adaptation of a mammalian muscle spindle. J Physiol. 1960;153(2):209-17.

32. Hunt CC, Ottoson D. Impulse activity and receptor potential of primary and secondary endings of isolated mammalian muscle spindles. J Physiol. 1975;252(1): 259-81.

33. Hodgkin AL, Huxley AF. The dual effect of membrane potential on sodium conductance in the giant axon of Loligo. J Physiol. 1952;116(4):497-506.

34. Moore P, Atema J. A model of a temporal filter in chemoreception to extract directional information from a turbulent odor plume. Bio Bull. 1988;174(3): 355-63.

35. Rushton DN. Functional electrical stimulation and rehabilitation - an hypothesis. Med Eng Phys. 2003; 25(1):75-8.

36. Schmidt R, Dudel J, Jänig W, Zimmermann M, Silva JFA. Neurofisiologia. São Paulo: EPU; 1979.
37. Torre V, Ashmore JF, Lamb TD, Menini A. Transduction and adaptation in sensory receptor cells. J Neurosci. 1995;15(12):7757-68.

38. Kandel ER, Jessell TM, Schwartz JH. Principles of neural science. 3rd ed. New York: Elsevier; 1991.

39. Guyton AC. Fisiologia humana. 6a ed. Rio de Janeiro: Guanabara Koogan; 1988.

40. Bahill T. Bioengineering - biomedical, medical, and clinical engineering. Englewood Cliffs, NJ: Prentice Hall; 1981.

41. Garcia EAC. Biofísica. São Paulo: Sarvier; 2002.

42. Malmivuo J, Plonsey R. Bioelectromagnetism, principles and applications of bioelectric and biomagnetic fields. New York: Oxford University Press; 1995.

43. Hodgkin AL, Huxley AF. Currents carried by sodium and potassium ions through the membrane of the giant axon of Loligo. J Physiol. 1952;116(4):449-72.

44. Baranauskas G. Ionic channel function in action potential generation: current perspective. Mol Neurobiol. 2007;35(2):129-50.

45. Hodgkin AL, Huxley AF, Katz B. Measurement of current-voltage relations in the membrane of the giant axon of Loligo. J Physiol. 1952;116(4):424-48.

46. Okuno E, Caldas IL, Chow C. Física para ciências biológicas e biomédicas. São Paulo: Harbra; 1986.

47. Hille B. Ionic channels in excitable membranes. Current problems and biophysical approaches. Biophys J. 1978;22(2):283-94.

48. Guyton AC, Esbérard CA, de Araujo CLC. Neurociencia básica: anatomia e fisiologia. 2a ed. Rio de Janeiro: Guanabara Koogan; 1993.

49. Fitzhugh R. Impulses and physiological states in theoretical models of nerve membrane. Biophys J. 1961; 1(6):445-66.

50. Bear MF, Connors BW, Paradiso MA. Neurociências: desvendando o sistema nervoso. 2a ed. Porto Alegre: Artmed; 2002.

51. Bean BP. The action potential in mammalian central neurons. Nat Rev Neurosci. 2007;8(6):451-65.

52. Baranauskas G, Martina M. Sodium currents activate without a Hodgkin and Huxley-type delay in central mammalian neurons. J Neurosci. 2006;26(2):671-84. 
53. Heneine IF. Biofísica básica. São Paulo: Atheneu; 2000.

54. Hodgkin AL, Huxley AF. The components of membrane conductance in the giant axon of Loligo. J Physiol. 1952;116(4):473-96.

55. Davies A, Blakeley AGH, Kidd C. Fisiologia humana. Porto Alegre: Artmed; 2002.

56. Chiu SY, Ritchie JM, Rogart RB, Stagg D. A quantitative description of membrane currents in rabbit myelinated nerve. J Physiol. 1979;292(1):149-66.

57. Alle H, Roth A, Geiger JRP. Energy-efficient action potentials in hippocampal mossy fibers. Science. 2009; 325(5946):1405-8.

58. Brandão ML. Psicofisiologia. São Paulo: Atheneu; 2000.

59. Sokolove PG, Cooke IM. Inhibition of impulse activity in a sensory neuron by an electrogenic pump. J Gen Physiol. 1971;57(2):125-63.

60. Huxley AF, Stämpfli R. Evidence for saltatory conduction in peripheral myelinated nerve fibres. J Physiol. 1949;108(3):315-39.

61. Rosenbluth J. A brief history of myelinated nerve fibers: one hundred and fifty years of controversy. J Neurocytol. 1999;28(4-5):251-62.

62. He JH. Resistance in cell membrane and nerve fiber. Neurosci Lett. 2005;373(1):48-50.

63. Collingridge GL. The induction of N-methyl-D-aspartate receptor-dependent long-term potentiation. Phil Trans R Soc Lond B. 2003;358(1432):635-41.

64. Machado ABM. Neuroanatomia funcional. 2a ed. São Paulo: Atheneu; 2006.

65. Barbara JG. Les heures sombres de la Neurophysiologie à Paris (1909-1939). La Lettre des Neurosciences. 2005;29:3-6.

66. Loewenstein WR, Mendelson M. Components of receptor adaptation in a Pacinian corpuscle. J Physiol. 1965;177(3):377-97.

67. Tolkacheva EG, Anumonwo JMB, Jalife J. Action potential duration restitution portraits of mammalian ventricular myocytes: role of calcium current. Biophys J. 2006;91(7):2735-45.

68. Deutsch S, Deutsch A. Understanding the nervous system: an engineering perspective. New York: WileyIEEE Press; 1993.
69. Fleidervish IA, Friedman A, Gutnick MJ. Slow inactivation of $\mathrm{Na}+$ current and slow cumulative spike adaptation in mouse and guinea-pig neocortical neurones in slices. J Physiol. 1996;493(Pt 1):83-97.

70. Peron S, Gabbiani F. Spike frequency adaptation mediates looming stimulus selectivity in a collisiondetecting neuron. Nat Neurosci. 2009;12(3):318-26.

71. Gandevia SC, Enoka RM, McComas AJ, Stuart DG, Thomas CK. Fatigue: neural and muscular mechanisms. New York: Springer US; 1995.

72. Powers RK, Sawczuk A, Musick JR, Binder MD. Multiple mechanisms of spike-frequency adaptation in motoneurones. J Physiol Paris. 1999;93(1-2):101-14.

73. Barrett EF, Barrett JN, Crill WE. Voltage-sensitive outward currents in cat motoneurones. J Physiol. 1980; 304(1):251-76.

74. Juusola M, French AS. Adaptation properties of two types of sensory neurons in a spider mechanoreceptor organ. J Neurophysiol. 1998;80(5):2781-4.

75. Reisert J, Matthews HR. Adaptation of the odourinduced response in frog olfactory receptor cells. J Physiol. 1999;519(3):801-13.

76. Juusola M, Seyfarth EA, French AS. Sodium-dependent receptor current in a new mechanoreceptor preparation. J Neurophysiol. 1994;72(6):3026-8.

77. Buller AJ. A model illustrating some aspects of muscle spindle physiology. J Physiol. 1965;179(3):402-16.

78. Brokensha G, Westbury DR. Adaptation of the discharge of frog muscle spindles following a stretch. J Physiol. 1974;242(2):383-403.

79. Buller AJ, Nicholls JG, Ström G. Spontaneous fluctuations of excitability in the muscle spindle of the frog. J Physiol. 1953;122(2):409-18.

80. Thomas F. Principles of electric circuits, electron flow version. 3rd ed. New York: Merrill; 1993.

81. Fanselow EE, Nicolelis MAL. Behavioral modulation of tactile responses in the rat somatosensory system. J Neurosci. 1999;19(17):7603-16.

82. Gorman RB, McDonagh JC, Hornby TG, Reinking RM, Stuart DG. Measurement and nature of firing rate adaptation in turtle spinal neurons. J Comp Physiol. 2005;191(7):583-603. 
83. Tian J, Iwasaki T, Friesen WO. Analysis of impulse adaptation in motoneurons. J Comp Physiol. 2010; 196(2):123-36.

84. Marini M, Veicsteinas A. The exercised skeletal muscle: a review. Eur J Translat Myology. 2010;20(3): 105-20.

85. Bellemare F, Woods JJ, Johansson R, Bigland-Ritchie B. Motor-unit discharge rates in maximal voluntary contractions of three human muscles. J Neurophysiol. 1983; 50(6):1380-92.

86. De Luca CJ, Forrest WJ. Some properties of motor unit action potential trains recorded during constant force isometric contractions in man. Kybernetik. 1973;12(3):160-8.

87. Gandevia SC. Neural control in human muscle fatigue: changes in muscle afferents, moto neurones and moto cortical drive. Acta Physiol Scand. 1998; 162(3):275-83.

88. Bigland-Ritchie B, Johansson R, Lippold OC, Smith $\mathrm{S}$, Woods JJ. Changes in motoneurone firing rates during sustained maximal voluntary contractions. J Physiol. 1983;340(1):335-46.
89. Kernell D, Monster AW. Time course and properties of late adaptation in spinal motoneurones of the cat. Exp Brain Res. 1982;46(2):191-6.

90. Kernell D, Monster AW. Motoneurone properties and motor fatigue. Exp Brain Res. 1982;46(2):197-204.

91. De Luca CJ, LeFever RS, McCue MP, Xenakis AP. Behaviour of human motor units in different muscles during linearly varying contractions. J Physiol. 1982; 329:113-28.

92. Spielmann JM, Laouris Y, Nordstrom MA, Robinson GA, Reinking RM, Stuart DG. Adaptation of cat motoneurons to sustained and intermittent extracellular activation. J Physiol. 1993;464(1):75-120.

Recebido: 22/02/2011

Received: 02/22/2011

Aprovado: 10/05/2011

Approved: 05/10/2011 